\title{
CURious Variables Experiment (CURVE): CCD photometry of active dwarf nova DI Ursae Majoris ${ }^{\star}$
}

\author{
A. Rutkowski ${ }^{1}$, A. Olech ${ }^{1}$, M. Wiśniewski ${ }^{1}$, P. Pietrukowicz ${ }^{1,2}$, J. Pala ${ }^{3}$, and R. Poleski ${ }^{4}$ \\ 1 Nicolaus Copernicus Astronomical Center, Polish Academy of Sciences, ul. Bartycka 18, 00-716 Warszawa, Poland \\ e-mail: [rudy; olech;mwisniew; pietruk]@camk.edu.pl \\ 2 Departamento de Astronomia y Astrofisica, Pontificia Universidad Catolica de Chile, Casilla 306, Santiago 22, Chile \\ 3 J. Pala's Amateur Observatory, Słupsk, Poland \\ 4 Warsaw University Observatory, Al. Ujazdowskie 4, 00-478 Warsaw, Poland
}

Received 30 October 2008 / Accepted 7 January 2009

ABSTRACT

\begin{abstract}
Context. We report an analysis of photometric behaviour of DI UMa, an extremely active dwarf nova. The observational campaign (completed in 2007) covers five superoutbursts and four normal outbursts.

Aims. We examined principal parameters of the system to understand peculiarities of DI UMa, and other active cataclysmic variables. Methods. Based on precise photometric measurements, temporal light curve behaviour, $\mathrm{O}-\mathrm{C}$ analysis, and power spectrum analysis, we investigated physical parameters of the system.

Results. We found that the period of the supercycle now equals $31.45 \pm 0.3$ days. Observations during superoutbursts infer that the period of superhumps equals $P_{\mathrm{sh}}=0.055318(11)$ days $(79.66 \pm 0.02 \mathrm{~min})$. During quiescence, the light curve reveals a modulation of period $P_{\text {orb }}=0.054579(6)$ days $(78.59 \pm 0.01 \mathrm{~min})$, which we interpret as the orbital period of the binary system. The values obtained allowed us to determine a fractional period excess of $1.35 \% \pm 0.02 \%$, which is surprisingly small compared to the usual value for dwarf novae $(2 \%-5 \%)$. A detailed $\mathrm{O}-\mathrm{C}$ analysis was performed for two superoutbursts with the most comprehensive coverage. In both cases, we detected an increase in the superhump period with a mean rate of $\dot{P} / P_{\mathrm{sh}}=4.4(1.0) \times 10^{-5}$.

Conclusions. Based on these measurements, we confirm that DI UMa is probably a period bouncer, an old system that reached its period minimum a long time ago, has a secondary that became a degenerate brown dwarf, the entire system evolving now toward longer periods. DI UMa is an extremely interesting object because we know only one more active ER UMa star with similar characteristics (IX Dra).
\end{abstract}

Key words. stars: individual: DI Ursae Majoris - stars: dwarf novae - stars: novae, cataclysmic variables - stars: binaries: close

\section{Introduction}

The ER UMa type stars (Kato \& Kunjaya 1995) remains one of the most intriguing systems among all cataclysmic variables. Those objects, belonging to SU UMa-type dwarf novae, exhibit various types of behaviour, such as normal outburst, superoutburst, and superhumps. Smak (1984) identified the origin of normal outbursts in dwarf novae in terms of thermal instability of the accretion disc. Whitehurst (1988) and Osaki (1989) then proposed a model that described superoutburst phenomena in those stars (for a review see Osaki 2005). The period between two consecutive superoutbursts (also called the supercycle period) in ER UMa type objects is about a few tens of days. Until now, the shortest known supercycle for this class of stars has been $P_{\mathrm{so}}=19.07$ days, the value measured for RZ LMi (Robertson et al. 1995; Olech et al. 2008).

DI UMa is a member of ER UMa class. The history of observations of DI UMa starts in 1959, when Hoffmeister (1959) discovered a rapidly varying star, which was later designated as DI UMa type. Further spectroscopical studies identified this object as a cataclysmic variable (Bond 1978; Szkody \& Howell 1992). Kato et al. (1996) determined the

* Photometric data are only available in electronic form at the CDS via anonymous ftp to cdsarc.u-strasbg.fr $(130.79 .128 .5)$ or via http://cdsweb.u-strasbg.fr/cgi-bin/qcat?J/A+A/497/437 period of the supercycle to be 25 days. Soon after, Fried et al. (1999) found that superoutbursts have a recurrence time of 30-45 days, and the superhump period and the orbital period equal 0.05529(5) and 0.054564(1) days, respectively. Hence, they measured the superhump period excess of $1.3 \%$, which was then one of the lowest for known CVs. Although a small deviation from regularity in the occurrence of superoutburst is admissible, this prominent change in supercycle length remains problematic from the standard thermal-tidal instability model point of view. This fact, in addition to a low period excess, that is appropriate for quiet WZ Sge stars, both with global properties and parameters characterizing this system, have encouraged us to take a closer look at the behaviour of DI UMa.

\section{Observations and data reduction}

Observations were carried out between January 15 and June 25 in 2007. Two telescopes were used to collect the data. The first was a 0.6-m Cassegrain telescope of Warsaw University Observatory located at its Ostrowik station. It is equipped with a Tektronix TK512CB back-illuminated CCD camera. The image scale was $0.76 /$ pixel providing a $6.5 \times 6.5$ field of view (Udalski $\&$ Pych 1992). The second was a f/3.8 0.3-m Newton telescope located at Jacek Pala's amateur observatory in Słupsk, equipped with a SBIG ST-2K dual CCD camera. 
Observations of DI UMa reported in this paper cover 36 nights and include 3093 measurements. The average exposure time was about $150 \mathrm{~s}$. Although we have not used an autoguider, images of stars remain unshifted and PSF profiles seem undistorted.

For the observational data processing, we used the IRAF ${ }^{1}$ package. The profile photometry was then obtained using the DAOphotII package (Stetson 1987). A standard way of reduction was performed for the Ostrowik data. Unfortunately, amateur observations from Słupsk were obtained without flat-field calibration images, so only simplified reduction was possible. This measurements are of poorer accuracy but sufficient to be included in the analysis.

Observations were carried out without filters for two reasons. First, due to the lack of an autoguiding system, we wished to maintain exposures as short as possible to minimize guiding errors. Second, because our main goal was an analysis of the temporal behaviour of the light curve, the use of filters could cause the object to become too faint to be observed in quiescence.

Relative unfiltered magnitudes of DI UMa were determined to be the difference between the magnitude of the variable and the magnitude of a nearby comparison star. Although we do not include AAVSO measurements in further analysis, we used these data to draw conclusions (from the overlap of two light curves) about the mean $V$ magnitude of the star. This procedure may introduce a relatively high systematic uncertainty in transforming the magnitudes, probably even as large as $\sim 0.3 \mathrm{mag}$ in the zero point.

Table 1 presents a journal of our CCD observations of DI UMa. In the following parts of this article (for convinience), civil dates are used to characterize moments of observations, rather than the corresponding HJD. In total, we observed the star for almost $113 \mathrm{~h}$.

\section{Global light curve}

Figure 1 presents the photometric behaviour of DI UMa during this campaign. The shape of the light curve corresponds to the standard picture of active dwarf novae (Olech et al. 2004b; Kato \& Kunjaya 1995; Robertson et al. 1995). Frequent superoutbursts reach $V \approx 14.5 \mathrm{mag}$ at maximum and fade to $V \approx 17.8$ at quiescence, so the amplitude of the superoutburst reaches $A_{\text {sup }} \approx 3.3$ mag. Between prominent superoutbursts, we can identify normal outbursts, reaching $\approx 15.4$ mag with an amplitude of $\approx 2.4$ mag.

The predictions of typical scenarios developed to explain the dwarf-novae light curve, in particular, its excess of brightness during superoutburst (compared to normal outburst brightness) as well as its duration time and profile, are in agreement with our observations. This allowed us to distinguish unambigously between measurements collected in different phases of activity. From the global light curve, we then selected only those nights during which the star was in superoutburst (marked by open circles). For the resulting light curve, we then computed the power spectrum using the ZUZA code of SchwarzenbergCzerny (1996).

The power spectrum was computed for a range of speeds $0-0.2 \mathrm{c} / \mathrm{d}$; the frequencies found to represent the longest periods that could be seen in the light curve and reflect the variability

\footnotetext{
${ }^{1}$ IRAF is distributed by the National Optical Astronomy Observatory, which is operated by the Association of Universities for Research in Astronomy, Inc., under cooperative agreement with the National Science Foundation.
}

Table 1. Observational journal for the DI UMa campaign.

\begin{tabular}{|c|c|c|c|c|c|}
\hline $\begin{array}{l}\text { Date in } \\
2007\end{array}$ & $\begin{array}{c}\text { Start [HJD] } \\
(2454000+)\end{array}$ & $\begin{array}{c}\text { End [HJD] } \\
(2454000+)\end{array}$ & $\begin{array}{l}\text { Dur } \\
\text { (h) }\end{array}$ & $\begin{array}{l}\text { No. of } \\
\text { points }\end{array}$ & $\begin{array}{c}\langle V\rangle \\
(\mathrm{mag})\end{array}$ \\
\hline Jan. 15 & 116.40818 & 116.64998 & 5.80 & 101 & 14.84 \\
\hline Mar. 07 & 167.33473 & 167.56941 & 5.63 & 97 & 16.23 \\
\hline Mar. 13 & 173.49266 & 173.65707 & 3.95 & 117 & 15.26 \\
\hline Mar. 26 & 186.33092 & 186.64037 & 7.43 & 99 & 17.69 \\
\hline Mar 27 & 187.32404 & 187.52248 & 4.76 & 89 & 16.95 \\
\hline Mar 28 & 188.33963 & 188.55087 & 5.07 & 106 & 15.71 \\
\hline Mar. 30 & 190.35326 & 190.46698 & 2.73 & 48 & 17.51 \\
\hline Mar. 31 & 191.36005 & 191.38821 & 0.68 & 9 & 17.69 \\
\hline Apr. 04 & 195.30920 & 195.31517 & 0.14 & 7 & 17.41 \\
\hline Apr. 05 & 196.33060 & 196.35222 & 0.52 & 24 & 15.73 \\
\hline Apr. 11 & 202.31427 & 202.34950 & 0.85 & 26 & 17.65 \\
\hline Apr. 12 & 203.34770 & 203.46420 & 2.80 & 79 & 17.46 \\
\hline Apr. 13 & 204.34525 & 204.47620 & 3.14 & 96 & 15.98 \\
\hline Apr. 14 & 205.36642 & 205.52808 & 3.88 & 115 & 14.70 \\
\hline Apr. 15 & 206.27831 & 206.47507 & 4.72 & 172 & 14.67 \\
\hline Apr. 16 & 207.28708 & 207.58366 & 7.12 & 523 & 14.82 \\
\hline Apr. 17 & 208.27757 & 208.40331 & 3.02 & 102 & 14.87 \\
\hline Apr. 18 & 209.34435 & 209.53969 & 4.69 & 123 & 14.93 \\
\hline Apr. 20 & 211.35228 & 211.46002 & 2.59 & 69 & 15.06 \\
\hline Apr. 21 & 212.28355 & 212.37567 & 2.21 & 61 & 15.21 \\
\hline Apr. 23 & 214.37639 & 214.46617 & 2.15 & 58 & 15.52 \\
\hline Apr. 25 & 216.28540 & 216.52020 & 5.64 & 176 & 17.15 \\
\hline Apr. 26 & 217.32268 & 217.44514 & 2.94 & 38 & 17.40 \\
\hline Apr. 29 & 220.39641 & 220.51590 & 2.87 & 22 & 16.08 \\
\hline May 16 & 237.44159 & 237.51015 & 1.65 & 42 & 14.77 \\
\hline May 17 & 238.33788 & 238.49019 & 3.66 & 139 & 14.83 \\
\hline May 18 & 239.33408 & 239.51366 & 4.31 & 136 & 14.94 \\
\hline May 20 & 241.36450 & 241.40883 & 1.06 & 24 & 15.11 \\
\hline May 21 & 242.36734 & 242.46793 & 2.41 & 63 & 15.22 \\
\hline May 22 & 243.38513 & 243.47040 & 2.05 & 52 & 15.30 \\
\hline May 23 & 244.37801 & 244.48001 & 2.45 & 63 & 15.30 \\
\hline May 24 & 245.34680 & 245.49097 & 3.46 & 66 & 15.61 \\
\hline May 25 & 246.33298 & 246.43703 & 2.50 & 26 & 16.66 \\
\hline Jun. 12 & 264.35449 & 264.36172 & 0.17 & 4 & 17.95 \\
\hline Jun. 19 & 271.37112 & 271.46188 & 2.18 & 53 & 14.72 \\
\hline Jun. 20 & 272.34845 & 272.41129 & 1.51 & 48 & 14.74 \\
\hline
\end{tabular}

related to superoutbursts. The resulting periodogram is shown in Fig. 2. Two prominent peaks can be observed. The highest peak at frequency of $0.0318 \mathrm{c} / \mathrm{d}$ corresponds to the period of the superoutburst cycle, which according to our measurements is 31.45 days. This value differs significantly from previous estimates made by Kato et al. (1996) and agrees with the range measured by Fried et al. (1999). Since, our light curve corresponds to 6 consecutive superoutbursts, the observational coverage is of higher quality than in earlier measurements and allowed us to achieve higher accuracies of period determinations.

As one can see from Fig. 1, the profiles of superoutbursts are fitted with solid lines. Those best-fit solutions were obtained for data belonging only to superoutburst IV, which had sufficient observational coverage. An average superoutburst profile was obtained by completing an analytical fit using Bezier curves. The derived profile was copied, in both forward and backward directions, with supercycle period of 31.45 days (as determined before), taking into account all acquired observational data. This line serves only as a check and demonstration tool and is not 


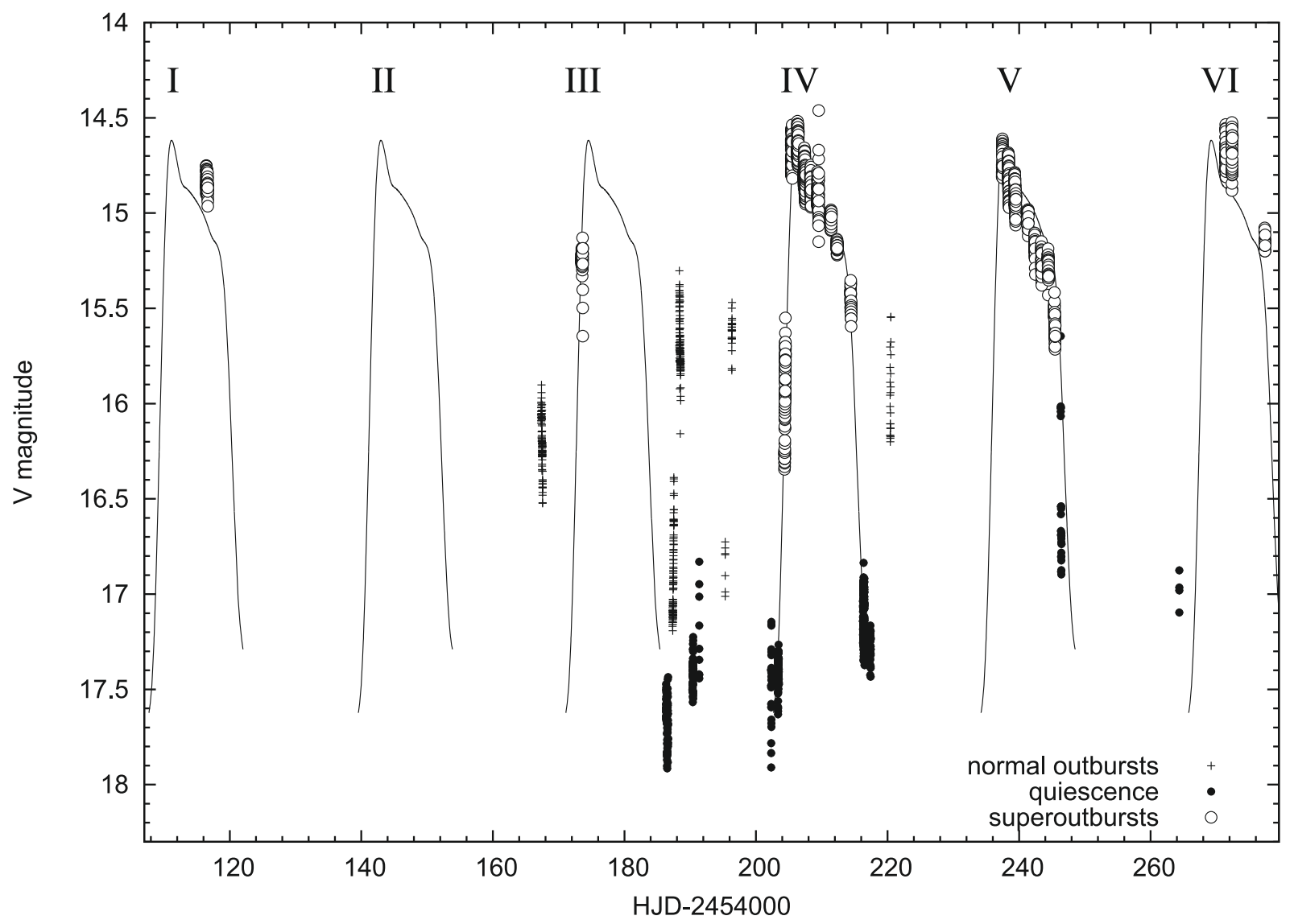

Fig. 1. Global light curve of DI UMa during the 2007 campaign. Dots, plus signs and circles indicate different periods of DI UMa activity. Those symbols represents quiescent state, normal outbursts and superoutbursts stage, respectively. Such division is used for further analysis (as in the text). Solid line shows a fit of the superoutburst profile No. IV, which is repeated every 31.45 days backward and forward.

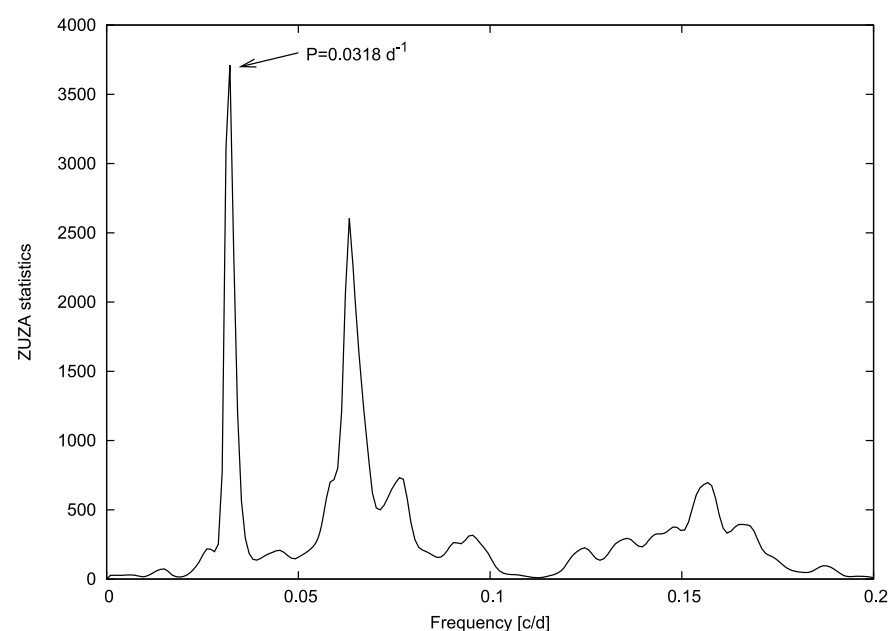

Fig. 2. Power spectrum of DI UMa light curve after removal of the data from quiescence and normal outbursts. The peak corresponding to the supercycle period is marked by an arrow.

used in later analysis, as for example in the detrending procedure described in Sect. 4.1. During the observing season, the stability of the supercycle was quite high, and the analytical light curve reproduce observations well.

Figure 3 presents the phased light curve folded with the supercycle period of $P_{\text {so }}=31.45$ days. As one can see, the shape of the superoutburst is typical. First, the brightness of the star increased rapidly from the $17.8 \mathrm{mag}$, during quiescent level, to

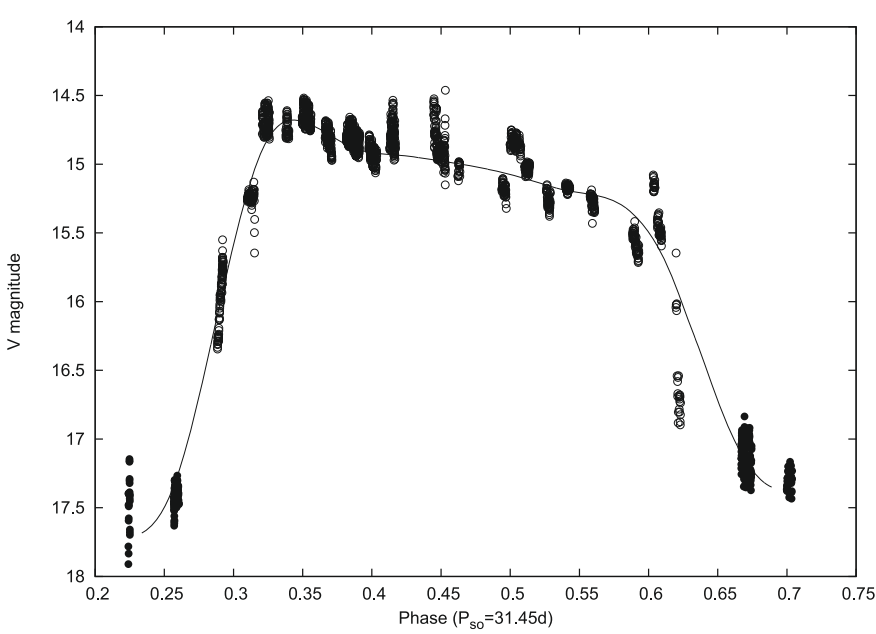

Fig. 3. Light curve of DI UMa in superoutbursts obtained by folding the general light curve with a supercycle period of 31.45 days.

a peak magnitude of around $14.5 \mathrm{mag}$. This increasing phase lasted $\sim 2$ days. A plateau with a slowly decreasing trend of rate $0.8 \mathrm{mag} / \mathrm{day}$, lasting $\sim 9.4$ days was observed. At the end of the plateau, the star reached $\sim 15.7 \mathrm{mag}$, then a far steeper decline occurred, and the final decline lasted $\sim 4$.1 days. Based on this figure, we conclude that on average the entire superoutburst lasts around $\approx 17.7$ days. In total, we have five certain detections of the superoutburst and one more (No. II) added as a prediction. 


\section{Superhumps}

Figure 4 shows the light curves of DI UMa during individual nights of the superoutburst that occurred in April 2007 (marked by roman number IV in Fig. 1). Data from twelve almost consecutive nights (gaps on Apr. 19, Apr. 22 and Apr. 24) are presented in this plot. One can clearly observe a variation in the superhumps from orbital humps (Apr. 12), a steep initial rise with a trace of modulation (Apr. 13), through fully-developed, toothshape, common superhumps (Apr. 14-21), to gradual disappearance and, finally, a transition to quiescence and orbital humps (Apr. 25-26). At maximum light, the amplitude of clearly visible common superhumps reaches almost 0.3 mag.

\subsection{Periodicity analysis}

As one may see from previous figures, the light curves of DI UMa are characterized by an alternating trend of increasing and decreasing and so on. Thus, the magnitudes had to be transformed to a common $V$ system. The fact that the data from quiescence to maximum of superoutbursts exhibited a variability in the range of about $3.3 \mathrm{mag}$, can have a pernicious effect on the period search. Taking this into acount, we prepared the data by subtracting the mean (and trend if necessary) from each night of time-series data as follows.

The data from each night were fitted with a straight line or a parabola and this fit was subtracted from the true light curve. This allowed us to remove the trend in all nightly light curves and shift the data from the entire campaign to a common level. As a result, the data have average brightness equal to zero and consist of only short-term modulations with periods significantly shorter than one day. After this approach, we expect an improvement in the reliability of the period determination.

One can expect that the power spectrum based on all available data can be contaminated by several smaller peaks and aliases due to the presence of various periodicities in the light curve. Thus, based only on one periodogram covering all available data, it is difficult to extract reliably all frequencies present in the light curve. For this reason, we decided to perform a more detailed frequency analysis.

\subsubsection{Superoutburst data analysis}

We consider again Fig. 1. We separated all points into three types of data. Points marked by open circles are measurements completed during the superoutburst phase. These points, after the removal of trends, were considered first in the analysis. The periodicity search was completed using the ZUZA code (Schwarzenberg-Czerny 1996).

The lowest panel of Fig. 5 presents the resulting periodogram. The most prominent peak correspond to the superhump period equal to $P_{\mathrm{sh}}=0.05504(5)$ days. This value is in agreement with the previous determination completed by Fried et al. (1999).

We also checked the period stability of superhumps during successive superoutbursts. Figure 5 shows a comparison of periodograms obtained for different superoutbursts. As one can see, there is no sign of a significant superhump period change from one superoutburst to another. Nevertheless, periodograms for different superoutbursts may be inconclusive due to insufficient data being available used in their preparation. There is only one night for the superoutburst No. I, and three nights for the superoutburst No. VI. For the same reason, aliases can distort significantly the obtained spectrum.
The light curve from which trends have been substracted that contain superhumps from all superoutbursts might be out of phase by an entire period exhibiting no significant evidence of phase shift between superhumps from different superoutbursts. Such a light curve phased with a period $P=0.05504$ days is presented in Fig. 6.

To check the stability of the phase of superhumps, we plot phased light curves separately for each night. We selected the superoutburst No. IV, for which we have data of the highest quaility coverage. Figure 7 presents the result of this approach. Successively, light curves from April 14 to 23 are shown from top to bottom in the plot. During the first four nights only, a slight or no phase-shift was observed. Later on (around Apr. 18), one can see the emergence of a secondary hump, although with out significant phase reversal, in contrast to some ER UMa stars (for example see Kato et al. 2003). One may expect that a modulation connected with the orbital period, present in a quiescent light curve, may also be detected in superoutbursts as in the case of another active dwarf nova, IX Dra (Olech et al. 2004a). This idea encouraged us to search for frequencies correlated with orbital humps in the power spectrum. We decided to perform prewhitening of the detrended light curve of DI UMa from all superoutbursts. First we removed from the light curve a modulation with a period corresponding to superhumps. We then performed a power spectrum analysis on the obtained data. We derived a noisy power spectrum with no significant peak, indicating that orbital modulation was absent during the superoutburst or its amplitude was below our detection limit.

\subsubsection{Quiescence data analysis}

We now consider the analysis of the data collected during quiescence (black dots in Fig. 1). We repeated the approach from Sect. 4.1.1 and produced a periodogram using the ZUZA software. Figure 8 presents the results of this procedure. One of the most prominent peaks present in the power spectrum is located at a frequency of $f_{\text {orb }}=18.323 \pm 0.005 \mathrm{c} / \mathrm{d}$ $\left(P_{\text {orb }}=0.054576(15)\right.$ days $)$. From a statistical point of view, it is possible that some other, lower, peak represents the true modulation present in the quiescent light curve of DI UMa. However we recall the result of Fried et al. (1999), who obtained photometrically the orbital period of DI UMa equal to $P_{\text {orb }}=0.054564(1)$ days, which is in excellent agreement with our determination. Hence, we assume the value of $P_{\text {orb }}=0.054576(15)$ days as the most probable for the orbital period of the binary. We phased our quiescence data based on this determination. The result of this procedure is shown in Fig. 9.

In addition, we collected data during normal outbursts, but due to an insufficient amount of measurements we were unable to derive useful conclusions for these data.

\subsection{The $O-C$ analysis}

DI UMa often exhibits clear and periodic modulation in quiescence, with an amplitude reaching even 0.4 mag. For this modulation, we determined 15 moments of maxima. A least-squares linear fit to these data gives the following ephemeris:

$\mathrm{HJD}_{\text {orb-max }}=2454186.341(4)+0.054580(7) \times E$,

which agrees within the errors with the determination based on the power spectrum described in the previous paragraph. The combination of both of these measurements gives us our final value of the orbital period of the binary, which is equal to $P_{\text {orb }}=$ $0.054579(6)$ days $(78.59 \pm 0.01 \mathrm{~min})$. 


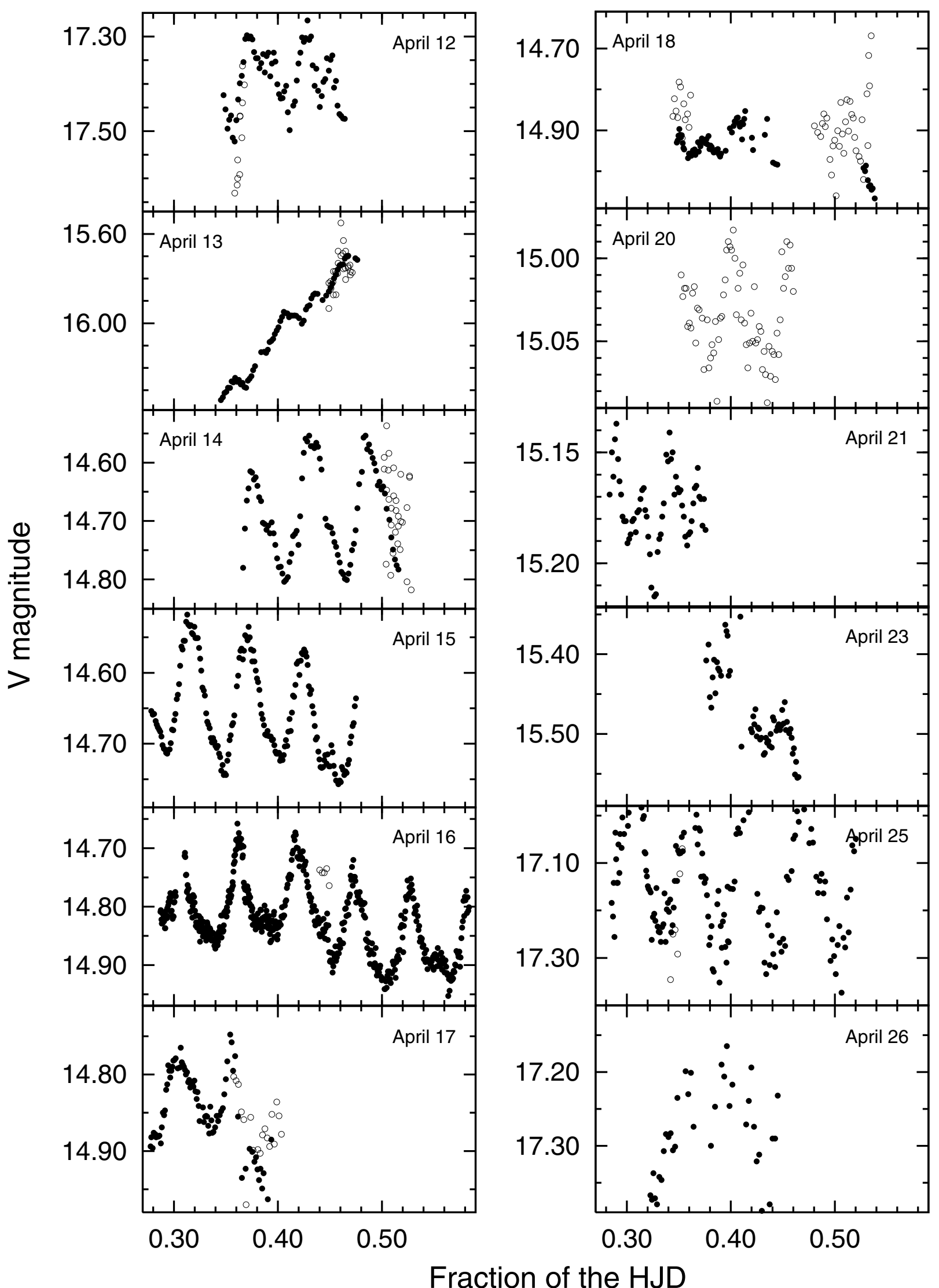

Fig. 4. Nightly light curves of DI UMa from its April 2007 superoutburst. Consecutive nights are denoted by dates in top-left/right corner. Dots shows the data obtained in Warsaw University Observatory, whereas open circles denotes observations made in Słupsk Observatory. 


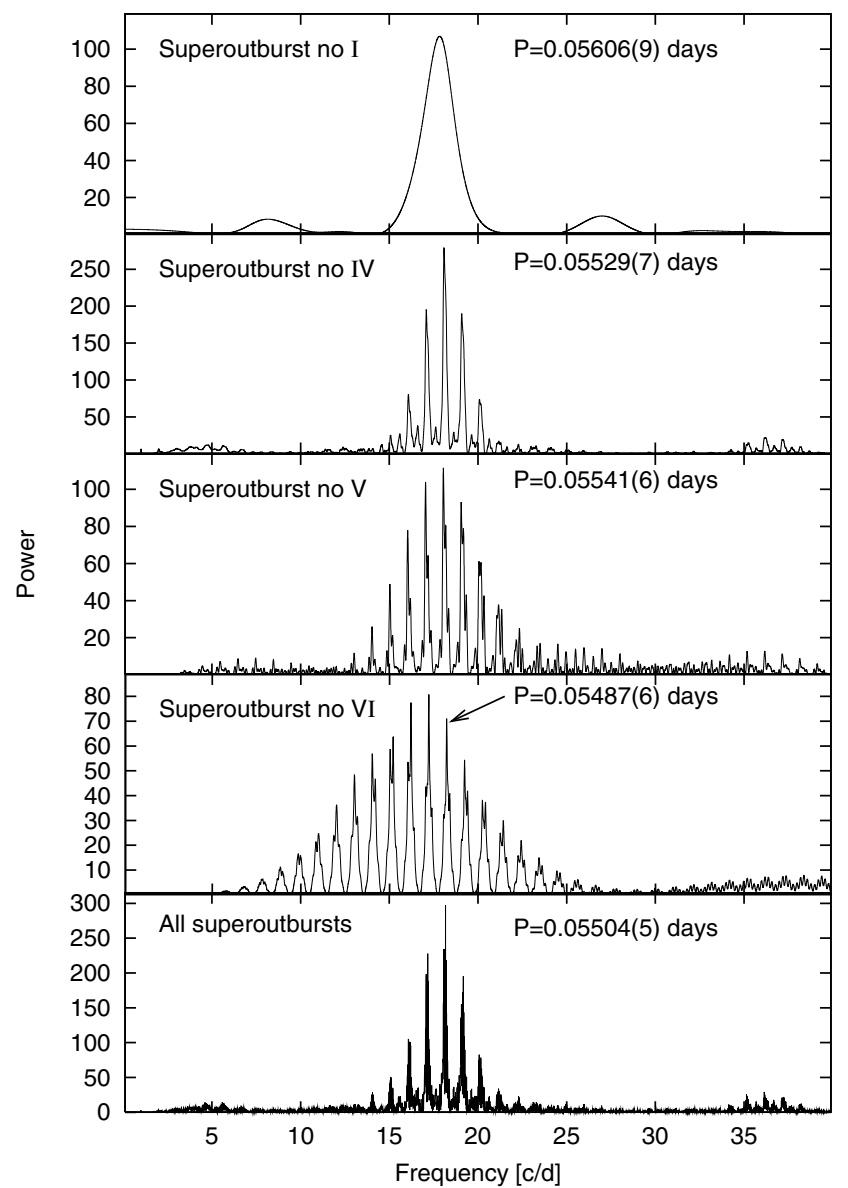

Fig. 5. Power spectra for superhumps observed during four superoutbursts of DI UMa. The numbers near the most prominent peaks represent the most probable value of superhump period.

On the other hand, the light curve of DI UMa from superoutbursts contains 38 moments of maxima.

The observations from superoutbursts Nos. IV and V contain sufficient data to perform a detailed analysis and draw valuable conclusions about the temporal evolution of the superhump period.

The maxima from superoutburst No. IV might be fitted with a common ephemeris in the form:

$\mathrm{HJD}_{\max }=2454205.3729(9)+0.055320(12) \times E$.

The $\mathrm{O}-\mathrm{C}$ values computed according to this ephemeris are shown in the top panel of Fig. 10. It is clear that they show a slightly increasing trend and thus we decided to fit the maxima with a quadratic ephemeris in the form:

$$
\begin{array}{r}
\mathrm{HJD}_{\max }=2454205.3761(12)+0.055139(50) \times E \\
+1.23(33) \cdot 10^{-6} \times E^{2} .
\end{array}
$$

We emphasize that the maxima denoted by negative cycle numbers relate to the night of April 12, i.e. from the rapid initial rise to the superoutburst, and were not included in the above fits. They are shifted in phase by about half a cycle and might not correspond to ordinary superhumps but to early superhumps or even orbital humps. The light curve for April 13 resembles the shape of early superhumps known from other studies (Kato 2002, Maehara 2007). Osaki \& Meyer (2002) proposed that early superhumps were caused by two-armed dissipation pattern on the acretion disc. During our observations, the early superhumps have a double-peaked profile and its amplitude was in

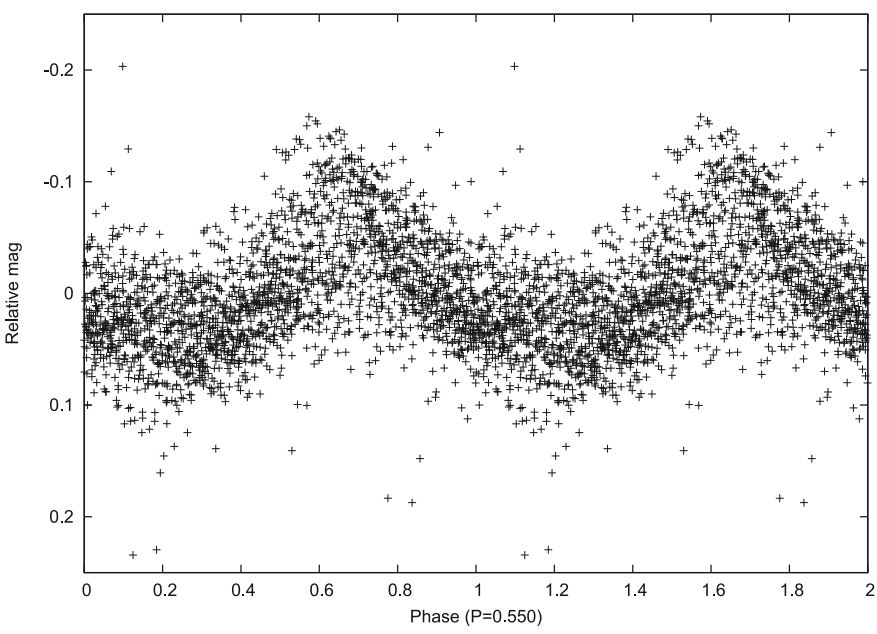

Fig. 6. Detrended light curve from data collected during all observed superoutbursts in 2007 campaign, folded with superhump period of $P_{\mathrm{sh}}=0.05504$ days.

the range of 0.05-0.07 mag. Unfortunately, since the distinctive shape of early sperhumps was visible only in April 13, the precise determination of its period was impossible. The crude estimate (read from graph) infers that $P_{\text {esh }} \cong 0.0540$ days with high uncertainty about $1 \%$. In light of previous observations of early superhumps (for example Patterson 2003), suggesting that $P_{\text {esh }}$ is equal to around orbital period, we consider this value with caution.

The maxima from superoutburst No. V can be fitted with a linear ephemeris of the form:

$\mathrm{HJD}_{\max }=2454237.46511(97)+0.055313(22) \times E$.

In this case, a slightly increasing trend in the superhump period was also observed, and the maxima could be fitted with a quadratic ephemeris, which is given by the following equation:

$$
\begin{array}{r}
\mathrm{HJD}_{\max }=2454237.4669(13)+0.055183(68) \times E \\
+1.18(58) \cdot 10^{-6} \times E^{2} .
\end{array}
$$

The superhump periods inferred from linear fits can be used together to compute our final mean value of the superhump period, which is $P_{\mathrm{sh}}=0.055318(11)$ days (79.66 $\left.\pm 0.02 \mathrm{~min}\right)$.

We note that period derivatives obtained during both superoutbursts are consistent within errors and provide a relatively low value of $\dot{P} / P_{\text {sh }}=4.4(1.0) \times 10^{-5}$.

We recall the figure from Uemura et al. (2005), which was updated by Rutkowski et al. (2007), showing the relation between the period derivative and the superhump period. It is presented in Fig. 11. The figure shows that DI UMa is placed in the group of small superhump periods $\left(P_{\mathrm{sh}}\right)$ and exhibits a low but positive $P / P_{\text {sh }}$ value.

\section{Conclusions}

Our five-month observational campaign concerning an active SU UMa-type star DI UMa resulted in the detection of five superoutbursts, which repeat every 31.45 days. This is far longer than the 25 days obtained by Kato et al. (1996) but falls within the range of 30-45 days observed by Fried et al. (1999).

Our observations indicate that DI UMa exhibits periodic, light modulations both in quiescence and during superoutbursts. The first of these modulations, with $P_{\text {orb }}=0.054579(6)$ days $(78.59 \pm 0.01 \mathrm{~min})$, is interpreted as the orbital period of the 


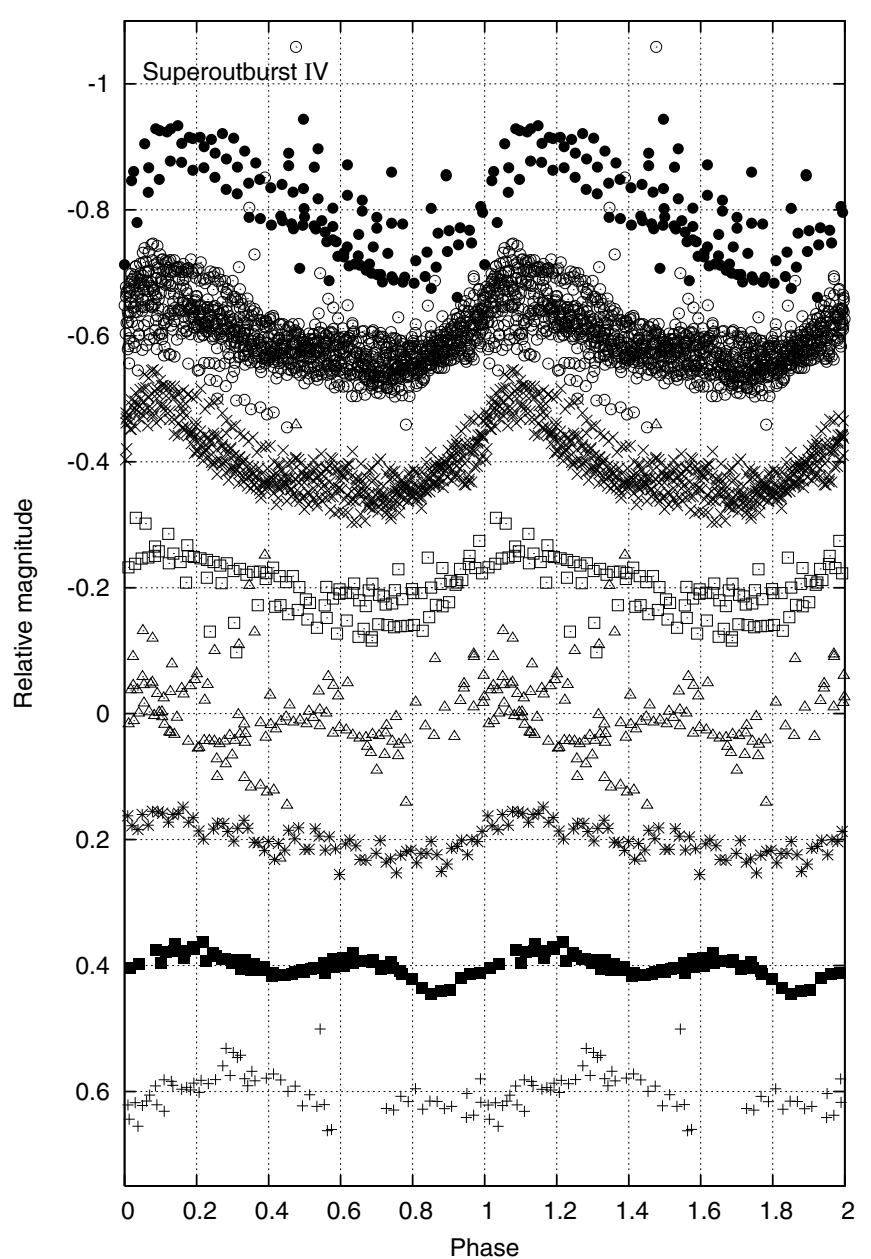

Fig. 7. Stability of superhumps phase during eight nights covering the April 2007 superoutburst, from April 14 (top to Apr. 23 (bottom). Light curves are phased with superhump period $P_{\mathrm{sh}}=0.05504$ days.

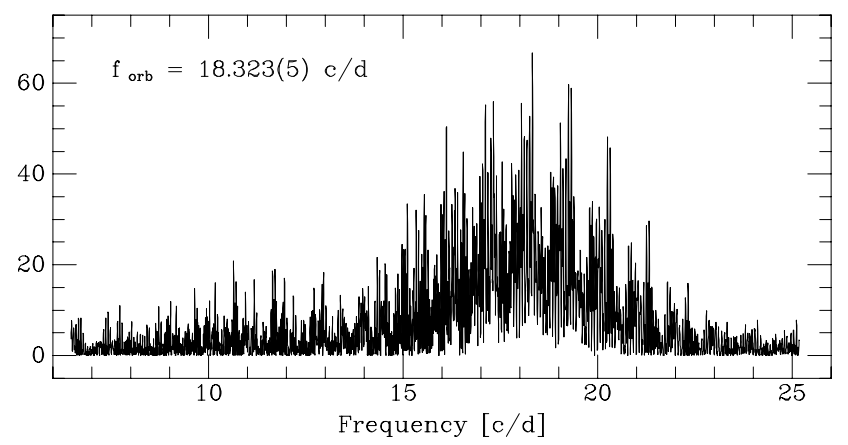

Fig. 8. Power spectrum of data collected during quiescence.

system and the second, with $P_{\mathrm{sh}}=0.055318(11)$ days $(79.66 \pm$ $0.02 \mathrm{~min}$ ), as the superhump period.

A very orbital period and a small period excess equal to only $1.35 \% \pm 0.02 \%$ suggest that DI UMa is a so-called period bouncer, i.e. an old system that reached its period minimum a long time ago, its secondary became a degenerated brown dwarf and the entire system now evolves toward longer periods (Patterson 2001). DI UMa is thus unique because we know only one more active ER UMa star with similar characteristics namely IX Dra (Olech et al. 2004a).

Inspection of Fig. 11 indicates that long-period systems tend to show large negative values of $\dot{P} / P_{\text {sh }}$, whereas short-period

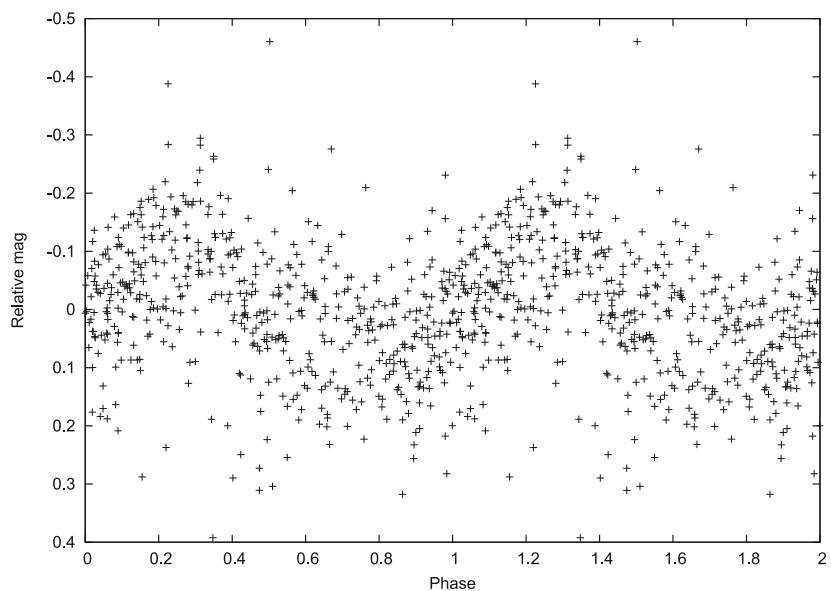

Fig. 9. Detrended light curve of data collected during quiescence and phased with period $P_{\text {orb }}=0.05458$ days, which we interpret as the orbital period of the system.

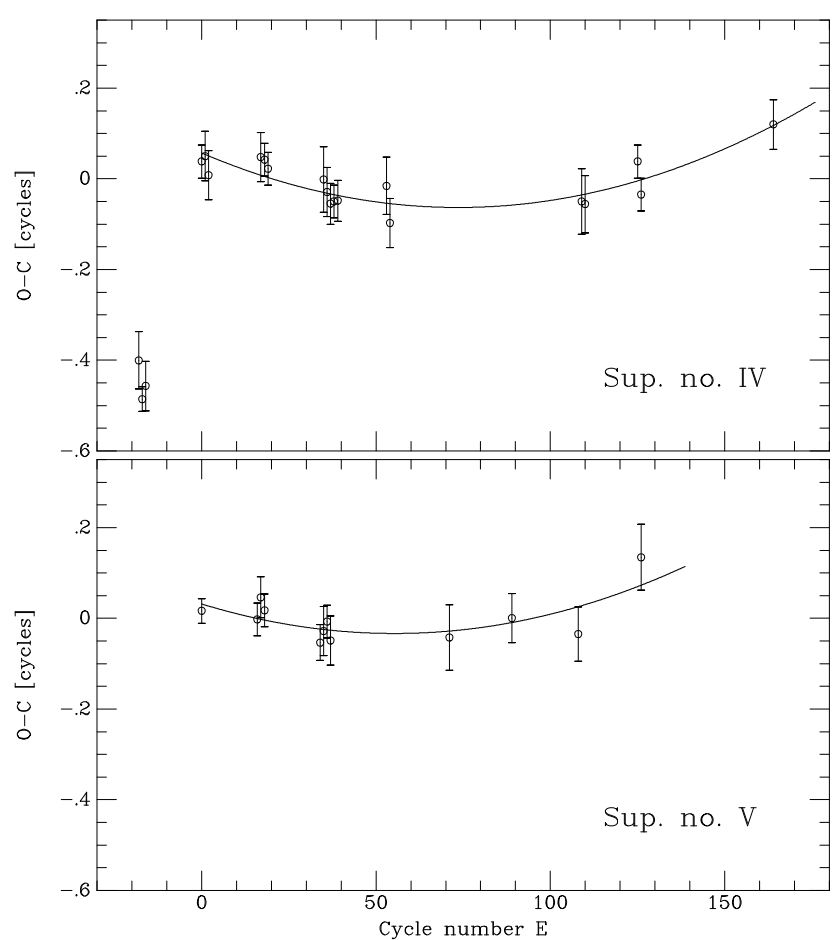

Fig. 10. O-C diagram for superhumps of DI UMa occurring during the April 2007 (IV) and May 2007 (V) superoutbursts. Solid lines correspond to quadratic ephemeris. Points with negative cycle numbers might be connected with orbital hump and not with superhumps.

systems are characterized by small and positive period derivatives. This picture has a physical interpretation proposed by Osaki \& Meyer (2003). In long-period dwarf-novae with high transfer rates, the 3:1 resonance radius in the accretion disc is close to the tidal truncation radius, and eccentric waves in the disc may thus propagate only inwards, proding a decrease in the superhump period. In short-period systems with lower accretion rates and more infrequent outbursts, the 3:1 radius is much smaller than the tidal truncation radius and sufficient matter is stored in the disc to cause the propagation of outward eccentric waves. In these systems, the superhump period may increase.

Do the properties of DI UMa inferred from observational data agree with this scenario? It is a short-period but active dwarf nova, showing both frequent outbursts and superoutbursts, which 


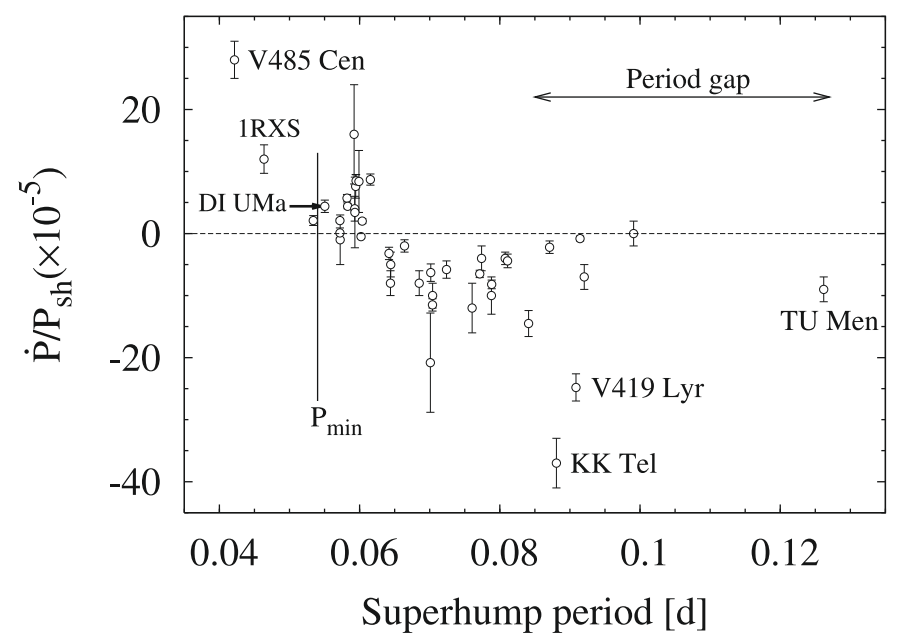

Fig. 11. Relation between the superhump period and its derivative for known SU UMa stars.

are indicate of a high-mass transfer-rate. It may imply that the disc contains sufficient matter to reach not only the $3: 1$ resonance radius, but also the region of the $2: 1$ resonance radius, as in the case of IX Dra, another active but old dwarf nova. There is thus a possibility of the ignition of outwardly propagating eccentric waves, which may cause an increase in the superhump period.

We recall that DI UMa is the second shortest-period dwarf nova $^{2}$ with a determined period derivative. Smaller values of both the superhump period and its derivative were determined for VS 0329+1250 (Shafter et al. 2007).

Acknowledgements. We acknowledge generous allocation of the Warsaw University Observatory 0.6-m telescope time. This work used the on-line service of the VSNET and AAVSO. We would like to thank Prof. Józef Smak for fruitful discussions. We also thank Maciej Bilicki for carefully reading this manuscript. This work was supported by MNiSzW grant No. N N203 301335 to A.O.

\section{References}

Bond, H. E. 1978, PASP, 90, 526

Fried, R. E., Kemp, J., Patterson, J., et al. 1999, PASP, 111, 1275

Hoffmeister, C. 1959, Astron. Nachr., 284, 275

Kato, T. 2001, PASJ, 53, L17

Kato, T. 2002, PASJ, 54, L11

Kato, T., \& Kunjaya, C. 1995, PASJ, 47, 163

Kato, T., Nogami, D., \& Baba, H. 1996, PASJ, 48, L93

Kato, T., Nogami, D., \& Masuda, S. 2003, PASJ, 55, L7

Maehara, H., Hachisu, I., \& Nakajima, K. 2007, PASJ, 59, 227

Nogami, D., Uemura, M., Ishioka, R., Kato, T., \& Pietz, J. 2004, PASJ, 56, 155

Olech, A. 1997, Acta Astron., 47, 281

Olech, A., Schwarzenberg-Czerny, A., Kedzierski, P., et al. 2003, Acta Astron., 53,175

Olech, A., Zloczewski, K., Mularczyk, K., et al. 2004a, Acta Astron., 54, 57

Olech, A., Cook, L. M., Złoczewski, K., et al. 2004b, Acta Astron., 54, 233

Olech, A., Wisniewski, M., Zloczewski, K., et al. 2008, Acta Astron., 58, 131

Osaki, Y. 1989, PASJ, 41, 1005

Osaki, Y., \& Meyer, F. 2002, A\&A, 383, 574

Osaki, Y., \& Meyer, F. 2003, A\&A, 401, 325

Osaki, Y. 2005, Proc. Jpn Acad. Ser. B, 81, 291

Patterson, J. 2001, PASP, 113, 736

Patterson, J., Thorstensen, J. R., Kemp, J., et al. 2003, PASP, 115, 1308 Robertson, J. W., Honeycutt, R. K., \& Turner, G. W. 1995, PASP, 107, 443

Rutkowski, A., Olech, A., Mularczyk, K., et al. 2007, Acta Astron., 57, 267

Shafter, A. W., Coelho, E. A., \& Reed, J. K. 2007, PASP, 119, 388

Schwarzenberg-Czerny, A. 1996, ApJ, 460, L107

Smak, J. 1984, Acta Astron., 34, 161

Stetson, P. B. 1987, PASP, 99, 191

Szkody, P., \& Howell, S. B. 1992, ApJS, 78, 537

Udalski, A., \& Pych, W. 1992, Acta Astron., 42, 285

Uemura, M., Kato, T., Ishioka, R., et al. 2002, PASJ, 54, L15

Uemura, M., Mennickent, R. E., Ishioka, R., et al. 2005, A\&A, 432, 261

Warner B. 1995, Cataclysmic Variable Stars (Cambridge University Press)

Whitehurst, R. 1988, MNRAS, 232, 35

\footnotetext{
2 There are two variables, V485 Cen (Olech 1997) and EI Psc (Uemura et al. 2002), which show far shorter periods of superhumps but their status as classical SU UMa systems is unknown.
} 